\title{
Poroelasticity and Poroplasticity at Large Strains
}

\author{
L. Dormieux' and S. Maghous ${ }^{1}$ \\ 1 CERMMO, ENPC, 6-8, avenue Blaise-Pascal, Champs-sur-Marne, 77455 Marne-la-Vallée, Cedex 2 - France \\ e-mail: dormieux@cermmo.enpc.fr, maghous@cermmo.enpc.fr
}

Résumé - Poroélasticité et poro-élastoplasticité en grandes déformations - Cet article examine quelques aspects de la formulation du comportement d'un milieu poreux saturé en évolution isotherme dans le domaine des transformations finies. Après avoir rappelé les résultats propres aux solides non poreux, on s'intéressera successivement aux comportements poroélastique et poro-élastoplastique.

Si la phase solide qui constitue le squelette présente un comportement élastique dans le domaine des grandes déformations, un passage micro-macro démontrera que l'énergie libre du squelette constitue un potentiel thermodynamique pour le comportement macroscopique. Les arguments de ce potentiel sont la déformation macroscopique du squelette ainsi que la porosité lagrangienne. Ces deux quantités s'interprètent commes des variables d'état macroscopiques. Dans le cas où le comportement du solide est élastoplastique en grandes déformations, on propose un cadre thermodynamique permettant de formuler une théorie de la poroplasticité finie.

Les techniques de changement d'échelle permettent de clarifier certains aspects de la formulation du comportement macroscopique. En particulier, la validité du concept de contrainte effective en poroélasticité et en poroplasticité finies est établie lorsque la phase solide est incompressible.

Même lorsque la déformation macroscopique imposée à un élément de volume de milieu poreux est infinitésimale, le champ de déformation à l'échelle microscopique peut être non infinitésimal. De ce fait, la simulation du comportement macroscopique de ce volume élémentaire dans le cadre d'un passage micro-macro doit être effectuée en tenant compte de transformations finies à l'échelle microscopique. Ce point est illustré par l'exemple du chargement œdométrique.

Mots-clés : porélasticité, poroplasticité, grandes déformations, approche micro-macro.

Abstract - Poroelasticity and Poroplasticity at Large Strains - This paper reviews some aspects of the formulation of the constitutive behavior of a saturated porous material in isothermal evolutions in the domain of large strains. First, the results concerning the nonporous solid are recalled. Then, the poroelastic and poro-elastoplastic behaviors at large strains are successively considered.

When the solid phase is elastic at large strains, a micro-macro approach shows that the free energy of the skeleton is a macroscopic thermodynamic potential. The latter depends on the macroscopic strain of the skeleton and on the lagrangian porosity, which can be interpreted as macroscopic state variables. When the solid is elastoplastic at large strains, a theory of finite poro-elastoplasticity is proposed within a macroscopic thermodynamic framework.

The homogenization techniques allow to clarify some aspects of the formulation of the macroscopic behavior. In particular, the validity of the effective stress principle in finite poroelasticity and poroplasticity is established when the solid phase is incompressible. Even if the macroscopic strain applied to an elementary volume is infinitesimal, the strain field at the microscopic scale may not be infinitesimal. Hence, the simulation of the macroscopic behavior of this elementary volume in the framework of a micro-macro approach must take into account possible large strains at the microscopic scale.

Keywords: poroelasticity, poroplasticity, large strains, micro-macro approach. 


\section{INTRODUCTION}

The purpose of this paper is to review some basic aspects of the formulation of a constitutive law at large strains. It is restricted to the isothermal evolutions of a fully saturated medium.

The easiest way to address this issue is certainly to begin with the monophasic case, that is, the case of a solid materia with no porosity. This is the situation which is encountered at the microscopic scale when the solid constituent of the porous medium is considered. We shall therefore introduce finite elasticity and finite plasticity, which could possibly be used as constitutive laws for this solid constituent.

Once the behavior of the solid constituent is known, homogenization techniques allow, at least theoretically, to simulate the behavior of the skeleton, defined as the macroscopic description of the porous solid. Finite elasticity and plasticity for the solid will respectively lead to finite poroelasticity and poroplasticity for the skeleton.

These extensions to the porous case of theories developed for the monophasic one require that the role played by the pore pressure be clarified. We shall examine one particular aspect of this question, namely the validity of the effective stress principle which is widely used in the domain of infinitesimal strains.

\section{KINEMATICS}

To begin with, we briefly recall some of the mathematical tools which are used for the description of kinematics at large strain. Obviously, this description is appropriate for both the microscopic and the macroscopic scales. The considered scale (micro or macro) is not yet specified.

Let $d \Omega_{0}$ be an elementary volume in the initial configuration which is transformed into $\mathrm{d} \Omega$ by the deformation gradient $f$. According to the definition of $f$, the elementary vector $\underline{\mathrm{d}}_{0}$ is transformed into $\underline{\mathrm{d}} M=f \cdot \underline{\mathrm{d}} M_{0}$. We note that $f$ is related to the gradient of the displacement field $\xi$ by:

$$
f=1+\nabla \underline{\xi}
$$

The relevant strain concept at large strain is the GreenLagrange strain tensor $\delta$. Considering two material vectors $\underline{\mathrm{d}} M_{0}$ and $\underline{\mathrm{d}}_{M_{0}^{\prime}}^{\prime}$ which are respectively transformed into $\underline{\mathrm{d} M}$ and $\underline{\mathrm{d}} M^{\prime}, \delta$ is defined by:

$2 \mathrm{~d} M_{0} \cdot \delta \cdot \mathrm{d} M_{0}^{\prime}=\mathrm{d} M \cdot \mathrm{d} M^{\prime}-\mathrm{d} M_{0} \cdot \mathrm{d} M_{0}^{\prime} \Rightarrow \delta=\frac{1}{2}\left({ }^{t} \mathrm{f} \cdot \mathrm{f}-1\right)(2)$

Using Equation (1) into Equation (2) yields:

$$
\delta=\varepsilon+\frac{1}{2}^{t} \nabla \underline{\xi} \cdot \nabla \underline{\xi} \text { with } \varepsilon=\frac{1}{2}\left(\nabla \underline{\xi}+{ }^{t} \nabla \underline{\xi}\right)
$$

It is the sum of the symmetrical part of the displacement gradient, which is the well-known linearized strain tensor, and of an additional term, which is of the second order with respect to $\nabla \xi$. In the situation of infinitesimal transformation, that is $|\nabla \xi| \ll 1$, it is therefore possible to approximate $\delta$ by $\varepsilon$. However, in the domain of large strains, $\varepsilon$ has no physical relevancy. This can be illustrated on the example of a pure rotation of angle $\theta$ around the $z$-axis. Given the matrix of the deformation gradient, one obtains:

$$
f=\left(\begin{array}{ccc}
\cos \theta & -\sin \theta & 0 \\
\sin \theta & \cos \theta & 0 \\
0 & 0 & 1
\end{array}\right) \Rightarrow\left\{\begin{array}{l}
\delta=0 \\
\varepsilon=\left(\begin{array}{ccc}
\cos \theta-1 & 0 & 0 \\
0 & \cos \theta-1 & 0 \\
0 & 0 & 0
\end{array}\right)
\end{array}\right.
$$

As expected for a rigid body motion, the strain described by the Green-Lagrange tensor is 0 , whereas the components of $\varepsilon$ vary between 0 and -2 as $\theta$ increases.

The symmetrical part $d$ of the gradient of the velocity field $\underline{v}$ is referred to as Eulerian strain rate. It is related to the Lagrangian strain rate $\dot{\delta}$ by:

$$
d=\frac{1}{2}\left(\operatorname{grad} \underline{\underline{v}}+{ }^{t} \operatorname{grad} \underline{v}\right)=f^{-1} \cdot \dot{\delta} \cdot f^{-1}
$$

For an infinitesimal transformation $(f \approx 1)$, Equation (5) yields the approximation $d \approx \dot{\delta}$. However, in the general case, $d$ is not a time derivative and it will be useful to replace it by the righthand side of Equation (5).

\section{MICROSCOPIC AND MACROSCOPIC DESCRIPTIONS OF THE TRANSFORMATION}

Let us now specify the definitions of the microscopic and macroscopic scales.

At the macroscopic scale, a representative elementary volume $\Omega_{t}$ (r.e.v.) located at the macroscopic point $\underline{z}$ is an infinitesimal part of a larger structure $S_{t}$ (Fig. 1). It can be considered as the superposition of the macroscopic particles respectively made of fluid and solid, all located at the same point $\underline{z}$. In other words, the different phases are not distinguished geometrically at this scale. The macroscopic particle made of solid will be referred to as skeleton particle. $\Omega_{0}$ denotes the initial configuration of the r.e.v. with respect to the transformation of the skeleton. From now on, the physical quantities defined at the macroscopic scale will be denoted by capital letters. Hence, $F, J=\operatorname{det} F=\left|\Omega_{t}\right| /\left|\Omega_{0}\right|$, $\Delta, D$ and $\Sigma$ are the macroscopic deformation gradient in the transformation of the skeleton, its Jacobian, the corresponding Green-Lagrange strain, the Eulerian strain rate and the Cauchy stress, respectively. The kinematic quantities are defined as in Equations (2) and (5). 


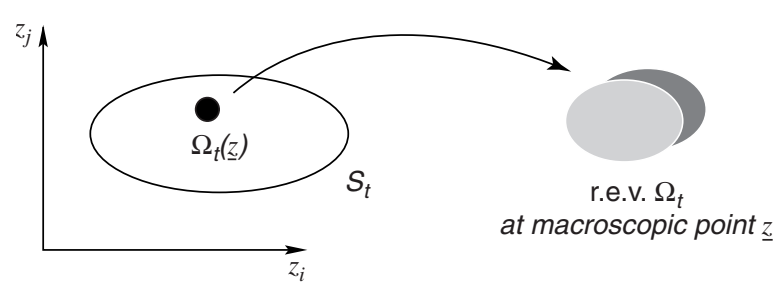

Figure 1

R.e.v. $\Omega_{t}$ at macroscopic scale.

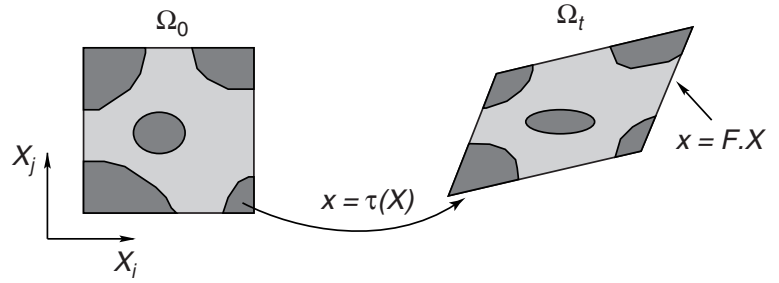

Figure 2

R.e.v. at microscopic scale: initial and deformed configurations.
According to the polar decomposition theorem, $F$ can be decomposed as the product of a rotation tensor $R$ and of a symmetrical pure stretch tensor $S$, the eigen values of which are the principal stretches:

$$
F=R \cdot S \text { with } S^{2}=1+2 \Delta
$$

At the microscopic scale, the solid and the fluid phases now occupy geometrically distinct domains $\Omega_{t}^{s}$ and $\Omega_{t}^{f}$ in $\Omega_{t}$ which should be regarded as a structure (Fig. 2).

The position vector is denoted by $\underline{x}$ in the deformed configuration $\Omega_{0}$ of the r.e.v. and by $\underline{X}$ in its initial configuration $\Omega_{0}$. The microscopic elementary volume in $\Omega_{0}$ and $\Omega_{t}$ are denoted by $\mathrm{d} \Omega_{0}$ and $\mathrm{d} \Omega_{t}$ respectively. Let $\underline{x}=\tau(\underline{X})$ be the transformation of the solid defined at the microscopic scale on the solid part $\Omega_{0}^{s}$ of $\Omega_{0}$. The condition $\Omega_{t}^{s}=\tau\left(\Omega_{0}^{s}\right)$ expresses the fact that the macroscopic skeleton particles in $\Omega_{0}$ and $\Omega_{t}$ contain the same solid particles at the microscopic level

The description of the transformation of the porous medium at the microscopic scale provided by $\tau$ can be related to the macroscopic deformation gradient $F$ by means of boundary conditions of the Hashin type (de Buhan et al., 1998). More precisely, the boundary $\partial \Omega_{t}$ of $\Omega_{t}$ is defined as the image of the boundary $\partial \Omega_{0}$ of $\Omega_{0}$ by the homogeneous transformation $\underline{x}=F \cdot \underline{X}$ associated with $F$. As far as the solid part of $\partial \Omega_{0}$ is concerned, this prescribes boundary conditions on the transformation $\tau$ :

$$
\text { on }\left(\partial \Omega_{0}\right)^{s}: \tau(\underline{X})=F \cdot \underline{X}
$$

The external boundary of the fluid domain $\Omega_{t}^{f}$ in the r.e.v. $\Omega_{t}$ is the image of the external boundary of the fluid domain $\Omega_{0}^{f}$ in the initial configuration by the homogeneous transformation $F \cdot \underline{X}$. However, as opposed to the case of the solid, $x=F \cdot \underline{X}$ is not the actual transformation of the fluid at the boundary $\partial \Omega_{0}$. Consequently, the fluid masses located inside the boundaries $\partial \Omega_{0}$ and $\partial \Omega_{t}$ respectively, as well as the corresponding macroscopic particles, are a priori different As a matter of fact, we shall see (Section 3.3) that the transformation of the fluid at the microscopic scale is not necessary for the determination of the overall behavior of the macroscopic skeleton particle.

We call Lagrangian porosity the ratio $\phi$ between the pore volume in the current configuration $\left|\Omega_{t}^{f}\right|$ and the initial total volume of the r.e.v.:

$$
\phi=\frac{\left|\Omega_{t}^{f}\right|}{\left|\Omega_{0}\right|}
$$

When one follows the transformation of the skeleton, the Lagrangian porosity $\phi$ is proportional to the pore volume. It will prove to be a convenient state variable in the formulation of the macroscopic behavior of the skeleton.

The physical quantities defined at the microscopic scale will be denoted by small letters. $f, j=\operatorname{det}(f), \delta, d$ and $\sigma$ denote the microscopic deformation gradient, its Jacobian, the Green-Lagrange strain, the Eulerian strain rate and the Cauchy stress, respectively:

$$
f=\nabla_{X} \tau ; \delta=\frac{1}{2}\left({ }^{t} f \cdot f-1\right)
$$

The descriptions of the kinematics at the microscopic and macroscopic scales are related by Equation (7). As regards stresses, the internal forces are represented at the macroscopic level by the stress tensor $\Sigma(\underline{z})$, and by the tensor field $\sigma(\underline{x})$ at the microscopic one. These two concepts are related according to an average rule:

$$
\sum=<\sigma(\underline{\mathrm{x}})>_{\Omega_{t}}=\frac{1}{\left|\Omega_{t}\right|} \int_{\Omega_{t}} \sigma(\underline{\mathrm{x}}) \mathrm{dV}
$$

\section{FINITE ELASTICITY AND POROELASTICITY}

Considering the microscopic scale first, the constitutive law of the solid constituent is formulated in the framework of finite elasticity (Sections 3.1 and 3.2). Then, moving to the macroscopic scale (Section 3.3), the overall behavior of the skeleton at large strain is shown to be poroelastic.

\subsection{Eulerian and Lagrangian Stress Tensors}

The formulation of the constitutive law requires to clarify which stress concept should be related to the strain. A simple example is proposed in order to show that, unlike the case of 
infinitesimal transformation, the usual Cauchy stress tensor $\sigma$ is not a function of the strain alone. Let us consider the following experiment performed on a cylindrical sample of the solid (Fig. 3). It is first loaded by two opposite forces $\pm F t$, where $t$ denotes a unit vector on the cylinder axis. This loaded configuration, denoted by $\kappa^{0}$, is taken as initial configuration. The Cauchy stress tensor $\sigma^{0}$ in $\kappa^{0}$ is of the form $\sigma \underline{t} \otimes \underline{t}$. Both the loading and the sample are then subjected to the same rotation $r$ (configuration $\kappa$ ). During this second stage of the loading, both strain rates $d$ and $\dot{\delta}$ are obviously equal to 0 whereas the Cauchy stress is given by:

$$
\sigma=\sigma(r \cdot \underline{t}) \otimes(r \cdot \underline{t})
$$

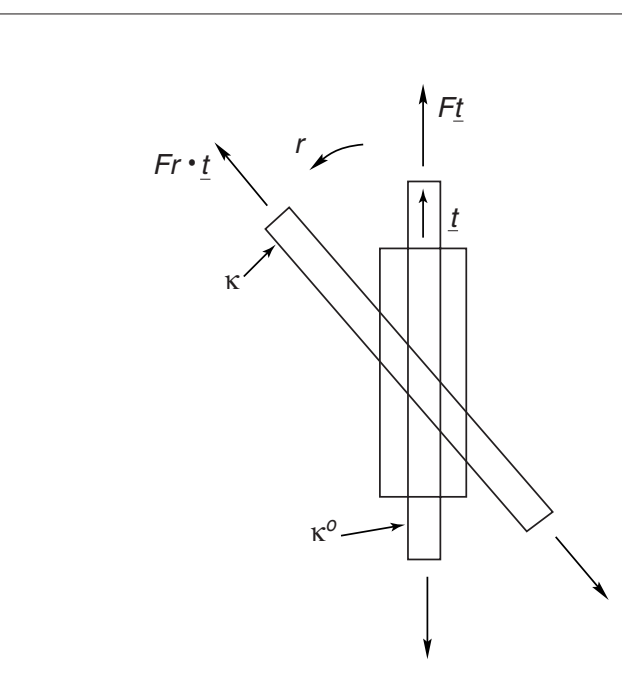

Figure 3

Evolution of the Cauchy stress during rotation.

which shows that the Cauchy stress $\sigma$ varies during the rotation, whereas $d=\dot{\delta}=0$. Hence, the constitutive law can obviously not be put in the form $\sigma=£(\delta)$. In other words, the Cauchy stress is not related to the strain alone.

In order to overcome this difficulty, we now introduce the Piola-Kirchhoff stress tensor $\pi$ wihch is defined on the initial configuration. For a given local deformation gradient $f$, an elementary material surface $\mathrm{d} S^{0}$ in the initial configuration and its image $\mathrm{d} S$ are related by $\mathrm{d} S=j^{t} f^{-1} \cdot \mathrm{d} S^{0}$. We define $\pi$ by the following condition: the Cauchy stress vector $\sigma \cdot \underline{\mathrm{d} S}$ is obtained by applying the deformation gradient $f$ to the PiolaKirchhoff stress vector $\pi \cdot \underline{\mathrm{d}}^{0}$.

$$
\sigma \cdot \underline{\mathrm{d} S}=f \cdot \pi \cdot \underline{\mathrm{d}}^{\mathrm{o}} \Rightarrow \pi=j f^{-1} \cdot \sigma \cdot{ }^{t} f^{-1}
$$

If we again consider the experiment described above in which the deformation gradient $f$ is a rotation $f=r$, Equation (12) shows that $\pi$ is a constant, equal to the Cauchy stress $\sigma$ in the initial configuration $\kappa^{0}$. This suggests that $\pi$ could be a relevant candidate for the formulation of the constitutive law under large strains.

\subsection{Finite Elasticity Applied to the Solid Constituent}

This is confirmed by the energy approach which can be performed in the case of an elastic solid. Let us consider an elementary volume $d \Omega_{0}$ in the initial configuration which is transformed into $\mathrm{d} \Omega_{t}$. The work developed by the internal forces in $\mathrm{d} \Omega_{t}$ is $P_{\text {int }}=\sigma: d \mathrm{~d} \Omega_{t}$. The combination of Equations (5) and (12) provides an alternative Lagrangian expression of $P_{\text {int }}$ wich involves the Lagragian stress and strain $\pi$ and $\delta$

$$
P_{i n t}=\sigma: d \mathrm{~d} \Omega_{t}=\pi: \dot{\delta} \mathrm{d} \Omega_{0}
$$

For an elastic solid, there is no dissipation. Hence, under isothermal evolution, the work of internal forces is entirely stored in the free energy of the solid. This thermodynamic function depends on a single state variable, namely the strain tensor $\delta$. It can be characterized by a Lagrangian density $\psi^{s}(\delta)$ wich represents the mechanical energy required to impose the strain $\delta$ to the unit volume of solid in the initial configuration. In term of rates, we thus have:

$$
\dot{\psi}^{s} \mathrm{~d} \Omega_{0}=\pi: \dot{\delta} \mathrm{d} \Omega_{0}
$$

The state equation of the elastic solid at large strain is immediatly derived from Equation (14):

$$
\pi=\frac{\partial \psi^{s}}{\partial \delta}(\delta)
$$

Introducing the relationship (12) between $\sigma$ and $\pi$ into Equation (15) yields an equivalent formulation of the constitutive law with respect to $\sigma$ :

$$
\sigma=\frac{1}{j} f \cdot \frac{\partial \psi^{s}}{\partial \delta}(\delta) \cdot{ }^{t} f=\boldsymbol{F}(\delta, r)
$$

As expected, Equation (16) shows that $\sigma$ is a function of the strain $\delta$ and of an additional parameter which is the rotation part $r$ of the deformation gradient decomposed as in Equation (6).

\subsection{Finite Poroelasticity}

We now move to the formulation of the constitutive law of the skeleton of a porous medium at the macroscopic scale. The skeleton particle of the r.e.v $\Omega_{t}$ is made of an elastic solid constituent, the state equation of which is given by Equation (15) at the microscopic level. The porous space is filled by a fluid at the uniform pressure $u$.

In the sequel, the constitutive law of the skeleton is derived from a homogenization technique. The method consists in defining a mechanical boundary value problem at the microscopic scale on the solid part $\Omega_{0}^{s}$ of the r.e.v., the loading parameters being the macroscopic deformation gradient $F$ and the pore pressure $u$. The solid/fluid interface in $\Omega_{t}$ is denoted by $I^{s f}$. 
The solid domain appears to be subjected to mixed boundary conditions, the displacement (Equation (7)) and the stresses being specified on $\left(\partial \Omega_{0}\right)^{s}$ and $I^{s f}$ respectively:

$$
I^{s f}: \sigma \cdot \underline{n}=-u \underline{n} ;\left(\partial \Omega_{0}\right)^{s}: \tau(\underline{\mathrm{X}})=F \cdot \underline{\mathrm{X}}
$$

The transformation $\tau$ of the solid is the solution of the problem defined by Equation (17) together with Equations (12) and (15). It depends on the loading parameters $F$ and $u$. Once $\tau$ is determined, the displacement of the solid/fluid interface $I^{f s}$ which is the internal boundary of the fluid domain is known. In addition, its external boundary has been defined by $F$. Consequently, the pore volume is known and the Lagrangian porosity can be considered as a function of $F$ and $u$. We assume that the relation $\phi(F, u)$ can be solved with respect to $u$ in the form $u=u(F, \phi)$. From now on, we shall consider that the couples of loading parameters $(F, u)$ and $(F, \phi)$ are equivalent.

This allows to consider $f=\nabla_{X} \tau$ as function of $\underline{X}, F$ and $\phi$ As for the strain field $\delta$, it is readily seen that it is not affected by the rotation part $R$ of $F$ introduced in Equation (6). $\delta$ and the microscopic Lagrangian density $\psi^{s}$ in $\Omega_{0}^{s}$ therefore appear as function of $\underline{X}, \Delta$ and $\phi$. By integration of $\psi^{s}$ over $\Omega_{0}^{s}$ we obtain the macroscopic free energy density $\Psi^{s k}$ of the skeleton:

$$
\Psi^{s k}(\Delta, \phi)\left|\Omega_{0}\right|=\int_{\Omega_{0}^{s}} \psi^{s}(\underline{\mathrm{X}}, \Delta, \phi) \mathrm{d} V
$$

Through Equation (18), it appears that $\Delta$ and $\phi$ control the value of the macroscopic free energy of the skeleton and can be therefore considered as macroscopic state variables.

The solid matrix being elastic, the work of the external forces applied to the solid part of the r.e.v. $P_{\text {ext }}$ is equal to the rate $\dot{\Psi}^{s k}\left|\Omega_{0}\right|$ of free energy. Using Equations (13) and (14), one obtains:

$$
P_{e x t}=\dot{\Psi}^{s k}\left|\Omega_{0}\right|=\int_{\Omega_{0}^{s}} \pi: \dot{\delta} \mathrm{d} V=\int_{\Omega_{t}^{s}} \sigma: d \mathrm{~d} V
$$

Rearranging the last integral in Equation (19) with Equations (8) and (10) yields:

$$
P_{e x t}=u \dot{\phi}\left|\Omega_{0}\right|+\Sigma: D\left|\Omega_{t}\right| \text { with } D=\left(\dot{F} \cdot F^{-1}\right)_{s}
$$

where $D$ is the macroscopic Eulerian strain rate. According to the definition (8), the term $u \dot{\phi}\left|\Omega_{0}\right|$ in Equation (20) can be interpreted as the work of the pore pressure in the pore volume change. $D$ appears as the macroscopic Eulerian strain rate in the transformation of the skeleton. Hence, $\Sigma: D\left|\Omega_{t}\right|$ represents the work of the macroscopic Cauchy stress $\Sigma$ in $\Omega_{t}$. In order to introduce a time derivative in this quantity, it is convenient to define a macroscopic Piola-Kirchhoff stress tensor $\Pi$, related to $\Sigma$ as in Equation (12). Besides, Equation (5) allows to replace $D$ by the Lagrangian strain rate $\dot{\Delta}$ :

$$
\Pi=J F^{-1} \cdot \Sigma \cdot{ }^{t} F^{-1} ; J \Sigma: D=\Pi: \dot{\Delta}
$$

A combination of Equations (19), (20) and (21) provides a Lagrangian expression of $\dot{\Psi}^{s k}$ :

$$
\dot{\Psi}^{s k}=u \dot{\phi}+\Pi: \dot{\Delta}
$$

from which we finally derive the macroscopic state equations of poroelasticity at large strain:

$$
\begin{gathered}
\Pi=\frac{\partial \Psi^{s k}}{\partial \Delta}(\Delta, \phi) \\
u=\frac{\partial \Psi^{s k}}{\partial \phi}(\Delta, \phi)
\end{gathered}
$$

Equation (23) can be interpreted as the macroscopic extension of Equation (15). In addition to the strain $\Delta$, the macroscopic state equations require a second state variable, namely the Lagrangian porosity $\phi$, which is necessary for the determination of both the Lagrangian stress tensor $\Pi$ and the pore pressure $u$.

However, special care is needed if the solid is incompressible. In this particular case, the Jacobian $J$ of the macroscopic deformation gradient $F$ is related to the Lagrangian porosity:

$$
J-\phi=1-\phi_{0}
$$

which expresses that the volume change of the skeleton particle is equal to the pore volume change. (25) thus implies that the state variables $\Delta$ and $\phi$ are not independent, so that the macroscopic free energy now appears as a function of $\Delta$ alone. More precisely, it can be shown from Equation (25) that:

$$
\dot{\phi}=\frac{\partial J}{\partial \Delta}: \dot{\Delta}=J F^{-1} \cdot{ }^{t} F^{-1}: \dot{\Delta}
$$

Replacing $\phi$ in Equation (22) by the above expression yields the single macroscopic state equation in the case of an incompressible solid:

$$
\dot{\Psi}^{s k}=\left(\Pi+u J F^{-1} \cdot{ }^{t} F^{-1}\right): \dot{\Delta} \Rightarrow \Pi+u J F^{-1} \cdot{ }^{t} F^{-1}=\frac{\partial \Psi^{s k}}{\partial \Delta}(\Delta)(27)
$$

Equation (27) introduces the Lagrangian effective stress $\Pi+u J F^{-1 . t}{ }^{t} F^{-1}$ which controls the value of the macroscopic strain $\Delta$. The combination of Equations (21) and (27) proves that the corresponding Eulerian effective stress is the usual Terzaghi's one:

$$
\Sigma^{\prime}(F)=\Sigma+u 1=\frac{1}{J} F \cdot \frac{\partial \Psi^{s k}}{\partial \Delta}(\Delta) \cdot{ }^{t} F
$$


Equation (28) constitutes an effective stress formulation of large strain poroelasticity: the deformation gradient $F$ is controlled by the value of the effective stress $\Sigma$ '. However, as opposed to the case of infinitesimal transformation, it should be noted that $\Sigma^{\prime}$ does not completely determine the strain of the skeleton if the rotation $R$ in Equation (6) is not given.

The above reasoning provides a generalization to large strains of Terzaghi's effective stress principle, however restricted to the case of an incompressible solid. It is likely that this assumption also constitutes a necessary condition as regards the possibility of formulating the macroscopic behavior in terms of Terzaghi's effective stress (de Buhan $e$ al., 1998).

\subsection{An Example of Micro-Macro Approach}

To finish with, let us present a very simple example in which the homogenization process can be carried out analytically The external boundary of the porous r.e.v. $\Omega_{0}$ is a vertical cylinder of axis $O Z$, external radius $R_{2}$ and height $\mathrm{H}_{0}$. The porous space $\Omega_{0}^{f}$ is a cylinder of same axis, of radius $R_{1}<R_{2}$. As before, $u$ denotes the pore pressure of the fluid filling the porous space. The constitutive solid is an incompressible neo-hookean material, for which the (microscopic) state equation writes:

$$
\sigma=\mu f \cdot{ }^{t} f+\eta 1
$$

$\eta$ is a nondetermined parameter which represents the Lagrange multiplier associated with the condition of incompressibility $j=1$, whereas $\mu$ is a material constant which generalizes the shear modulus of the standard Hooke's law. Indeed, the linearized form of Equation (29), which is:

$$
\sigma=2 \mu \varepsilon+\eta 1
$$

is the standard isotropic constitutive law for an incompressible linear elastic solid.

We consider the response of this r.e.v. to vertical compaction under the condition of an oedometer test: there is no lateral displacement at the boundary $R=R_{2}$ whereas the top of the cylinder $\left(Z=H_{0}\right)$ is subjected to a downwardsoriented vertical displacement $d=\alpha H_{0}$. In other words, the macroscopic deformation gradient of the r.e.v. is:

$$
F=\underline{e}_{X} \otimes \underline{e}_{X}+\underline{e}_{Y} \otimes \underline{e}_{Y}+(1-\alpha) \underline{e}_{Z} \otimes \underline{e}_{Z}
$$

However, for the sake of simplicity, the condition (7) in the planes $Z=0$ and $Z=H_{0}$ is replaced by:

$$
\left\{\begin{array}{l}
Z=0: \quad \tau_{z}=0 \\
Z=H_{0}: \tau_{z}=H_{0}(1-\alpha)
\end{array} ;\left\{\begin{array}{l}
z=0: \\
z=H_{0}(1-\alpha)
\end{array}: \sigma_{x z}=\sigma_{y z}=0\right.\right.
$$

The solid being incompressible, the generalized effective stress principle holds. According to Equation (28), it states that the vertical effective stress $\Sigma_{z z}^{\prime}=\sum_{z z}+u$ is controlled by $\alpha$. This is confirmed by the analytical resolution of the boundary value problem (de Buhan, 1998), which provides an expression of the force $Q(\alpha, u)$ applied on the top $(z=$ $\left.H_{\mathrm{o}}(1-\alpha)\right)$ of the r.e.v.:

$$
\begin{aligned}
& \Sigma^{\prime}{ }_{v}=\left|\Sigma_{z z}^{\prime}\right|=\frac{Q}{\pi R_{2}^{2}}-u=-\mu\left(\left(1-\phi_{0}\right)-\right. \\
& \left.\left(1-\alpha-\frac{1}{(1-\alpha)^{2}}-\frac{\alpha}{2(1-\alpha)^{2}\left(\phi_{0}-\alpha\right)}\right)+\frac{1}{2(1-\alpha)^{2}} \log \frac{\phi_{0}-\alpha}{\phi_{0}(1-\alpha)}\right)^{(33)}
\end{aligned}
$$

As expected, it can be shown that the linearization of $\Sigma^{\prime}$ 。 given by Equation (33) for $\alpha \ll \phi_{0}$ is equal to the solution of the linearized problem in which the constitutive law (29) has been replaced by Equation (30):

$$
\Sigma_{v}^{\prime \prime \operatorname{lin}}=\mu\left(1-\phi_{0}\right)\left(3+\frac{1}{\phi_{0}}\right) \alpha
$$

$\Sigma_{v}^{\prime}\left(\right.$ resp. $\left.\Sigma_{v}^{\prime l i n}\right)$ represents the macroscopic response of the r.e.v., as predicted by a nonlinear (resp. linear) homogenization process. As opposed to the linear analysis, the nonlinear one incorporates both the nonlinearity of the constitutive law (Eq. (29)) and the nonlinearity associated with geometry change. $\Sigma_{v}^{\prime}$ and $\Sigma_{v}^{\prime \prime \text { lin }}$ are plotted together against $\alpha=d / H_{0}$ at Figure 4 for an initial porosity $\phi_{0}=0.2$.

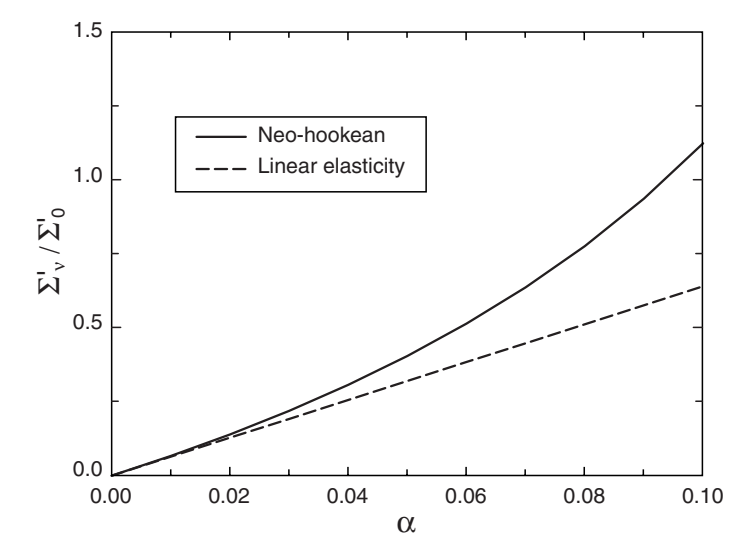

Figure 4

Macroscopic behavior: linear and non-linear micro-macro approaches.

Even if the macroscopic strain applied to the r.e.v. remains reasonably small, we observe that the discrepancy between the fully nonlinear and the linear analyses can be very significant, with a ratio $\Sigma^{\prime}{ }_{v} / \sum^{\prime}{ }_{v}$ lin of the order of 2 for $\alpha \approx 0.1$. This is due to the fact that the existence of a cavity in the r.e.v. introduces high heterogeneities in the microscopic strain field $\delta$, with a high strain level in the vicinity of the cavity. This effect increases as the porosity decreases. Hence, even if the r.e.v. is subjected to an infinitesimal transformation 
at the macroscopic scale, the microscopic transformation can be noninfinitesimal. In this case, the simulation of the macroscopic behavior by means of a micro-macro approach must obviously be performed within the framework of large strains.

\section{FINITE PLASTICITY AND POROPLASTICITY}

We now move to the elastoplastic behavior. Section 4.1 addresses the case of the solid at the microscopic scale. Section 4.2 examines the formulation of the skeleton constitutive law (macroscopic scale) in the light of a micromacro reasoning. Section 4.3 considers the same question within a purely macroscopic thermodynamic framework.

\subsection{Finite Plasticity Applied to the Solid Constituent}

\subsubsection{Elastoplasticity in Infinitesimal Transformation}

In the case of infinitesimal deformations, the linearized strain tensor $\varepsilon$ of an elastoplastic material is usually written as the sum of an elastic part $\varepsilon^{e}$ and a plastic part $\varepsilon^{p}$ :

$$
\varepsilon=\varepsilon^{e}+\varepsilon^{p}
$$

The same decomposition holds for the strain rates $d \approx \dot{\varepsilon}$ :

$$
d=d^{e}+d^{p}
$$

The strain which is observed when the load applied to the elementary volume is removed, is equal to $-\varepsilon^{e}$. Hence $\varepsilon^{p}$ can be interpreted as the residual strain in the unloaded configuration (Fig. 5).

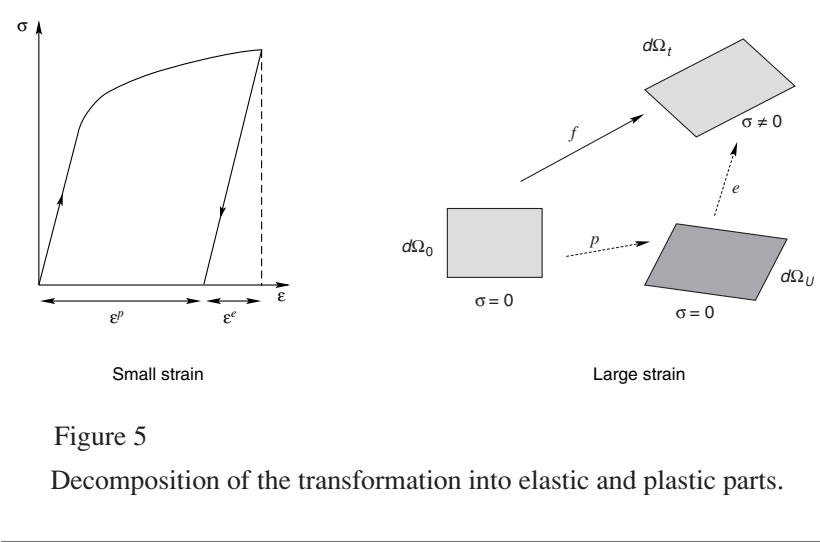

The state equation relates the Cauchy stress $\sigma$ and the elastic strain $\varepsilon^{e}$ :

$$
\sigma=\frac{\partial \psi^{s}}{\partial \varepsilon^{e}}
$$

where $\psi^{s}$ denotes as before the density of free energy in the solid. For linear elastic properties defined by the tensor $A$ of elastic moduli, Equation (37) simply takes the form:

$$
\sigma=A: \varepsilon^{e}
$$

In order to complete the formulation of the elastoplastic constitutive law, the flow rule must be specified. It determines the value of the plastic strain rate $d^{p}$ by means of the concept of plastic potential denoted here by $g(\sigma)$ :

$$
d^{p}=\dot{\lambda}\left(\frac{\partial g}{\partial \sigma}\right) \text { with } \dot{\lambda} \geq 0
$$

where $\dot{\lambda}$ is the plastic multiplier. For linear elastic properties, the combination of the state equation (38) and the flow rule (39) provides a simple relationship between the stress and strain rates:

$$
\dot{\sigma}=A: \dot{\varepsilon}^{e}=A:\left(d-\dot{\lambda} \frac{\partial g}{\partial \sigma}\right)
$$

\subsubsection{Decomposition of the Transformation - State Equation}

In the case of large strains, we want to keep the idea that the deformation gradient $f$ has a plastic and an elastic components, the plastic part being defined as the residual deformation gradient which remains when the load applied to the elementary volume is removed. Following Lee (1969), we therefore introduce the concept of unstressed configuration, denoted by $\mathrm{d} \Omega_{U}$, which is obtained by unloading from the deformed configuration $\mathrm{d} \Omega_{t}$. We assume that unloading and reloading between $\mathrm{d} \Omega_{t}$ and $\mathrm{d} \Omega_{U}$ are reversible. The elastic part $e$ of $f$ is defined as the deformation gradient which transforms $\mathrm{d} \Omega_{U}$ into $\mathrm{d} \Omega_{t}$. The plastic part $p$ is the deformation gradient which transforms $\mathrm{d} \Omega_{0}$ into $\mathrm{d} \Omega_{U}$. Hence, the generalization of Equation (35) to large strains writes:

$$
f=e \cdot p
$$

We assume that the solid is plastically incompressible, which implies that det $p=1$. In addition, we assume that the elastic strain $\delta^{e}$ is infinitesimal. In other words, large strains are of irreversible (plastic) nature only. This means that the elastic deformation gradient $e$ defines a reversible infinitesimal transformation:

$$
e=1+\varepsilon^{e} \text { with }\left|\varepsilon^{e}\right|<<1
$$

Let $j^{e}=\left|\mathrm{d} \Omega_{t}\right| /\left|\mathrm{d} \Omega_{U}\right|$ be the elastic jacobian and $\pi=$ $j^{e} e^{-1} \cdot \sigma \cdot{ }^{t} e^{-1}$ be the Piola-Kirchhoff stress tensor defined on the unstressed configuration associated with the Eulerian stress $\sigma$ 
applied to $\mathrm{d} \Omega_{t}$. If the free energy density of the solid in the unstressed configuration were a function $\psi^{s}\left(\delta^{e}\right)$ of the elastic strain $\delta^{e}$, we could expect that the generalized form of Equation (37) writes:

$$
\pi=\frac{\partial \psi^{s}}{\partial \delta^{e}} \text { with } \delta^{e}=\frac{1}{2}\left({ }^{t} e \cdot e-1\right)
$$

But, in the general case, this assumption $\Psi^{s}=\Psi^{s}\left(\delta^{e}\right)$ is not correct (Mandel, 1971). The origin of the difficulty lies in the fact that the unstressed configuration is not defined in a unique way.

As a matter of fact, the previous decomposition of the deformation gradient $f$ does not completely determine its elastic and plastic parts because the unloading process from $\mathrm{d} \Omega_{t}$ does not prescribe the orientation of the unstressed configuration. More precisely, given an arbitrary rotation tensor $v$ and a particular decomposition $\left(e^{*}, p^{*}\right)$ of $f$ associated with the unstressed configuration $\mathrm{d} \Omega_{U}^{*}$, the couple $\left(e=e^{*} \cdot{ }^{t} v\right.$ $\left.p=v \cdot p^{*}\right)$ is another possible decomposition. The corresponding unstressed configuration $\mathrm{d} \Omega_{U}$ is deduced from $\mathrm{d} \Omega_{U}^{*}$ by the rotation $r$. The elastic Green-Lagrange strain tensors $\delta_{*}^{e}$ and $\delta^{e}$ are related by $\delta^{e}=v \cdot \delta_{*}^{e} \cdot{ }^{t} v$.

The free energy of the solid in $\mathrm{d} \Omega_{t}$ can be characterized by a density defined with respect to the unstressed configuration. The numerical value of the density is obviously independen of the choice of the unstressed configuration whereas the state variables do depend on it. For instance, let us assume that the free energy density associated with the unstressed configuration $\mathrm{d} \Omega_{U}^{*}$ is a function $\delta_{*}^{e}$ of the elastic strain $\delta_{*}^{e}$. This implies that the free energy density $\psi^{s}$ associated with the unstressed configuration $\mathrm{d} \Omega_{U}^{*}$ depends on both the elastic strain $\delta^{e}$ and the orientation of $\mathrm{d} \Omega_{U}^{*}$ with respect to $\mathrm{d} \Omega_{U}^{*}$ defined by $r$. Eventually, the state equation (43) is valid only for the choice $\mathrm{d} \Omega_{U}^{*}$ in the form $\pi^{*}=\partial \psi_{*}^{s} / \partial \delta_{*}^{e}$.

In order to avoid this difficulty, we shall now assume that the elastic properties of the solid are isotropic. In this case, the elastic free energy depends only on the eigen values of the elastic strain. Hence, if the assumption $\psi_{*}^{s}=\psi_{*}^{s}\left(\delta_{*}^{e}\right)$ is valid for a particular orientation of the unstressed configuration, it is also valid for any other possible choice. For any orientation, the state equation (43) is now correct. From now on, we choose the particular unstressed configuration for which e is a symmetrical tensor, which determines its orientation in a unique way. This consists in putting the rotation component of the deformation gradient $f$ into the plastic part.

Equation (43) can be simplified with the assumption (42) of small elastic strain:

$$
\left\{\begin{array}{l}
\pi=j^{e} e^{-1} \cdot \sigma \cdot{ }^{t} e^{-1} \approx \sigma \\
\delta^{e} \approx \varepsilon^{e}
\end{array} \Rightarrow \sigma=\frac{\partial \psi^{s}}{\partial \varepsilon^{e}}\right.
$$

For instance, for linear elastic (isotropic) properties, Equation (44) takes the same form as in Equation (38):

$$
\sigma=A: \varepsilon^{e}
$$

However, there are two important differences between Equations (38) and (45).

First, as opposed to the elastic strain $\varepsilon^{e}$ which appears in Equation (38), the strain $\varepsilon^{e}=e-1$ in Equation (45) is not defined on the initial configuration but on a configuration deduced from the initial one by $p$ which is a priori a noninfinitesimal transformation. For instance, let us again consider the experiment described in Section 3.1, with the assumption that the constitutive behavior is linear elastic. In this example, $p$ is equal to the rotation $r$ whereas $\varepsilon^{e}$ is the infinitesimal elastic strain tensor induced by the couple of forces $\pm F r \cdot \underline{t}$ applied to the inclined sample after it has been subjected to the rotation:

$$
\varepsilon^{e}=\sigma A^{-1}:(r \cdot \underline{t} \otimes r \cdot \underline{t})
$$

The second difference is related to the characterization of the elastic evolutions. Within the infinitesimal framework, a possible definition of such an evolution is $\varepsilon=\varepsilon^{e}$ whereas the condition $\delta=\varepsilon^{e}$ is meaningless, the tensors $\delta$ and $\varepsilon^{e}$ being not defined on the same configuration. In fact, the characteristic property of an elastic evolution is that the unstressed configuration is subjected to a pure rotation which means that no plastic strain takes place. This can be expressed by the fact that the strain rate associated with the plastic component is 0 :

$$
\left(\dot{p} \cdot p^{-1}\right)_{s}=0
$$

where $(X)_{s}\left(\operatorname{resp} .(X)_{a}\right)$ denotes the symmetrical (resp. antisymmetrical) component of tensor $X$.

\subsubsection{Flow Rule}

We now address the question of the flow rule at large strain. First, a decomposition of the strain rate tensor $d$ is derived from Equation (41):

$$
d=\left(\dot{f} \cdot f^{-1}\right)_{s}=\left(\dot{e} \cdot e^{-1}\right)_{s}+\left(e \cdot \dot{p} \cdot p^{-1} \cdot e^{-1}\right)_{s}
$$

The two terms of the righthand side of Equation (48) depend on $\dot{e}$ and $\dot{p}$ respectively. For this reason, they are sometimes referred to as elastic and plastic strain rates. But this terminology can be misleading in so far as it suggests to write the flow rule on the second term, namely $\left(e \cdot \dot{p} \cdot p^{-1} \cdot e^{-1}\right)_{s}$. If it were true, this tensor should be equal to 0 in an elastic evolution of the elementary volume. However, such an evolution is characterized by Equation (47) which does not, in general, imply that $\left(e \cdot \dot{p} \cdot p^{-1} \cdot e^{-1}\right)_{s}=0$. 
A natural way to overcome this difficulty consists in writing the flow rule directly on the symmetrical part of the plastic strain rate:

$$
\left(\dot{p} \cdot p^{-1}\right)_{s}=\dot{\lambda} \frac{\partial g}{\partial \sigma} \text { with } \dot{\lambda} \geq 0
$$

Equation (49) appears as a simple extension of Equation (39) to large strains, and obviously complies with the requirement that the plastic multiplier $\dot{\lambda}$ should be equal to 0 in an elastic evolution. The plastic incompressibility can be expressed through the fact that the plastic potential $g$ in Equation (49) only depends on the deviatoric part of the Cauchy stress $\sigma$ so that $\partial g / \partial \sigma$ is a deviatoric tensor:

$(\forall \lambda) g(\sigma+\lambda 1)=g(\sigma) ; \frac{\partial g}{\partial \sigma}(\sigma+\lambda 1)=\frac{\partial g}{\partial \sigma}(\sigma) ; \operatorname{tr} \frac{\partial g}{\partial \sigma}=0(50)$

Accordingly, Equation (49) implies that the plastic transformation induces no volume change at the microscopic level.

\subsubsection{Relationship between Stress and Strain Rates}

Due to the fact that $\varepsilon^{e}$ is not defined on a fixed configuration, the state equation (45) is not very convenient. For practical purposes, it is useful to extend to large strain the relationship between stress and strain rates given in Equation (40) fo infinitesimal elastoplastic evolutions. In the case of a pure rotation, the righthand side in Equation (40) is equal to 0 , whereas $\dot{\sigma} \neq 0$ (Section 3.1). We thus expect that the generalized form of Equation (40) introduces a correcting term taking the effect of large rotation into account.

Introducing the assumption of small elastic strain (42) and the state equation (45) into Equation (48), the following expression of the Cauchy stress rate is derived:

$$
\left\{\begin{array}{l}
\dot{\sigma}=A:\left(d-\left(e . \dot{p} \cdot p^{-1} \cdot e^{-1}\right)_{s}\right) \\
\left(e \cdot \dot{p} \cdot p^{-1} \cdot e^{-1}\right)_{s} \approx\left(\dot{p} \cdot p^{-1}\right)_{s}+\varepsilon^{e} \cdot \dot{p} \cdot p^{-1}-\dot{p} \cdot p^{-1} \cdot \varepsilon^{e}
\end{array}\right.
$$

Using the state equation (45) again, as well as the flow rule (49) in Equation (51) yields the following relationship:

$$
\frac{D \sigma}{D t}=A:\left(d-\dot{\lambda} \frac{\partial g}{\partial \sigma}\right) \text { with } \frac{D \sigma}{D t}=\dot{\sigma}+\sigma \cdot \Omega-\Omega \cdot \sigma
$$

where $\Omega$ is the plastic rate of rotation $\left(\dot{p} \cdot p^{-1}\right)_{a}$. Within the framework of small elastic strain, it is readily seen that $\Omega$ can be approximated by the total rate of rotation $\left(\dot{f} \cdot f^{-1}\right)_{a}$.

The corresponding expression of $D \sigma / D t$ is the so-called Jaumann derivative. It can be interpreted as the time derivative of the Cauchy stress tensor $\sigma$ with respect to a rotating frame, having a rate of rotation $\Omega$. For instance, it is possible to characterize the evolution of the Cauchy stress in a pure rotation by $D \sigma / D t=0$ and not by $\dot{\sigma} \neq 0$, as we know from Section 3.1. As opposed to the conventional time derivative $\dot{\sigma} \neq 0$, it is therefore possible to relate the
Jaumann derivative of the Cauchy stress to the total and plastic strain rates.

We note that the extension of the state equation (40) to large strain only requires to add the correcting term $\sigma \cdot \Omega-\Omega \cdot \sigma$ in order to take into account the effects of noninfinitesimal rotation (on this very debated question, see also for instance Dienes (1979) and Gilormini (1994))

\subsection{Micro-Macro Approach for an Elastoplastic Solid}

\subsubsection{Validity of the Effective Stress Principle}

We now try to determine some aspects of the macroscopic constitutive law of the skeleton, the solid constituent at the microscopic scale being of the type described at section 4.1. As in 3.3, the macroscopic response is determined through the resolution of a mechanical problem defined on the solid part $\Omega_{0}^{s}$. The boundary conditions are of the same type as in Equation (17).

As before, the solid is plastically incompressible. In addition, in order to justify Terzaghi's effective stress principle (at the macroscopic scale), we assume that it is elastically incompressible. It is readily seen that the elastic incompressibility introduces an additional non determined term $\dot{\eta} 1$ in the state Equation (52), the origin of which is the same as in Equation (29):

$$
\dot{\sigma}+\sigma \cdot \Omega-\Omega \cdot \sigma=2 \mu\left(d-\dot{\lambda} \frac{\partial g}{\partial \sigma}\right)+\dot{\eta} 1
$$

Let $\sigma_{0}(t)$ and $\xi_{0}(t)$ respectively be the Cauchy stress and displacement fields in the solid domain which satisfies the boundary conditions (17) and the state Equation (53) in the particular case $u=0$, for a given evolution $t \rightarrow F(t)$ of the macroscopic deformation gradient, denoted by $\boldsymbol{F} \cdot(\boldsymbol{F}, u=0)$ will be referred to as the drained loading. $d_{0}$ and $\Omega_{0}$ denote the Eulerian strain rate and rotation rate associated with $\underline{\xi}_{0}(t) . \dot{\lambda}_{0}$ and $\dot{\eta}_{0}$ are the plastic and Lagrange multipliers in drained conditions. The macroscopic Cauchy stress tensor $\Sigma_{0}$ in a drained evolution is related to the stress field $\sigma_{0}$ according to Equation (10). As opposed to the elastic case (see Section 3.3), $\Sigma_{0}(t)$ depends (through $\sigma_{0}(t)$ ) not only on the current value $F(t)$ of the macroscopic deformation gradient at time $t$, but on the whole history $\boldsymbol{F}$ of the loading:

$$
\Sigma_{0}(\boldsymbol{F})=\frac{1}{\left|\Omega_{t}\right|} \int_{\Omega_{t}^{s}} \sigma_{0} \mathrm{~d} V
$$

We now superpose an arbitrary evolution $t \rightarrow u(t)$ of the pore pressure, denoted by $u$, on the evolution $\boldsymbol{F}$ of the deformation gradient. We introduce the stress field $\sigma(t)=\sigma_{0}(t)-$ $u(t) 1$ on the solid domain. Using the property stated in Equation (50), its Jaumann derivative writes:

$$
\frac{D \sigma}{D t}=\frac{D \sigma_{0}}{D t}+\dot{u} 1=2 \mu\left(d_{0}-\dot{\lambda}_{0} \frac{\partial g}{\partial \sigma}(\sigma)\right)+\left(\dot{u}+\dot{\eta}_{0}\right) 1
$$


Equation (55) proves that $\sigma(t)$ and $\xi_{0}(t)$ satisfy the state equation whereas they obviously comply with the boundary conditions (17) associated with $(\boldsymbol{F}, \boldsymbol{u})$. Hence, they are solutions of the corresponding mechanical problem on the solid domain.

We observe that the displacement is the same as in the drained case, so that the geometry of the r.e.v. $\Omega_{t}$ is not affected by the pore pressure. With respect to the drained case, the Cauchy stresses in the solid (and the fluid) are modified by addition of a uniform hydrostatic stress $-u 1$. According to Equation (10), this property of the local stress fields at the microscopic level is also valid at the macroscopic scale: the macroscopic Cauchy stress $\Sigma$ associated with the evolution $(\boldsymbol{F}, \boldsymbol{U})$ is very simply related to $\Sigma_{0}$ by:

$$
\Sigma=\Sigma_{0}(\boldsymbol{F})-u 1
$$

Equation (56) states that the macroscopic effective stress $\Sigma+u 1$ only depends on the deformation gradient history $\boldsymbol{F}$ and not on the pore pressure. This result proves that Terzaghi's effective stress principle remains valid when the solid constituent is subjected to finite elastoplastic transformations, provided that it is incompressible.

\subsubsection{A Numerical Example}

As in Section 3.4, the geometry at the microscopic level is now specified in order to simulate the overall behavior of a porous structure. The porous space is a horizontal cylindrical cavity which crosses the sample. The macroscopic deformation gradient is the same as in Equation (31). For the sake of simplicity, the boundary conditions slightly diffe from Equation (7). On the vertical lateral boundary, it is assumed that there are no shear stress and no normal displacement. At the top and bottom of the r.e.v., the boundary conditions are the same as in Equation (32). The pore pressure $u$ is applied on the solid/fluid interface.

The solid constituent is perfectly elastoplastic and incompressible, of the type described in Section 4.2.1. Therefore, Terzaghi's effective stress principle holds. The yield criterion is the von Mises' one. Due to the in compressibility of the solid, the volume of the porous space will decrease as compaction proceeds according to:

$$
\phi=\phi_{0}-\alpha
$$

We therefore expect that $\Sigma_{v}^{\prime}=\left|\Sigma_{z z}^{\prime}\right|$ becomes infinite when $\phi$ tends towards 0 . Three different numerica simulations of the macroscopic compaction curve are presented in Figure 6.

For a given level of macroscopic compaction characterized by $\alpha$, the highest estimate of the macroscopic vertica effective stress $\Sigma_{v}=\left|\Sigma_{z z}^{\prime}\right|$ is obtained in the fully nonlinear

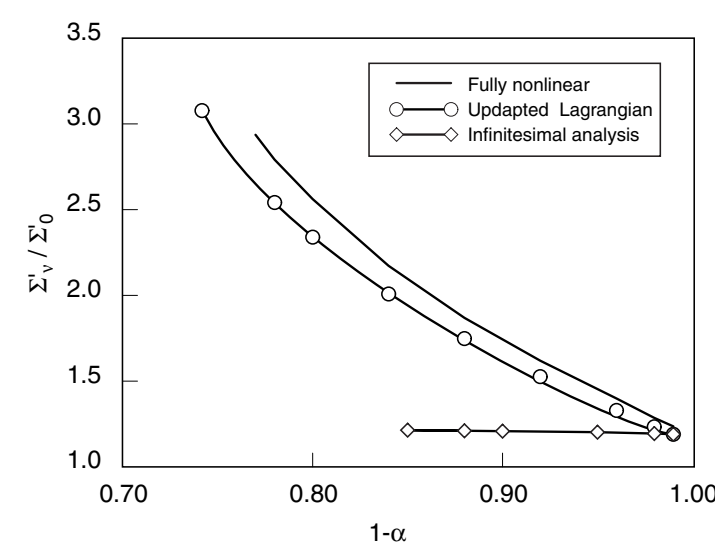

Figure 6

Simulations of the macroscopic compaction curve for an elastoplastic solid.

analysis which is based on state equation (53) and takes into account the change of geometry in the r.e.v. induced by the compaction. If the correcting term $\sigma \cdot \Omega-\Omega \cdot \sigma$ in Equation (53) is neglected but the change of geometry is taken into account according to an updated Lagrangian procedure, the estimate of $\Sigma_{v}^{\prime}$ is slightly smaller than the fully nonlinear one. This small discrepancy suggests that the effects of local large rotations within the r.e.v. are not of primary importance, at least within the range of macroscopic compaction covered by Figure 6. As expected, these simulations of the macroscopic compaction curve both show a strong increase of $\Sigma_{v}^{\prime}$ for increasing values of $\alpha$. A completely different pattern is obtained in the framework of small strain plasticity, according to which $\Sigma_{v}^{\prime}$ reaches an asymptote. Such a result is obviously not compatible with the incompressibility of the solid.

The comparison of the three simulations points out that geometry changes at the microscopic level control the macroscopic response. As a matter of fact, for a perfectly elastoplastic solid, we can interpret the vertical effective stress corresponding to the current compaction level as the limit load in the sense defined in limit analysis. For a given value of $\alpha$, this limit load depends on the shape of the cavity and can be determined by the usual methods of limit analysis. Excellent agreement between a kinematic estimate of the limit load and the fully nonlinear compaction curve presented in Figure 6 has been obtained by de Buhan et al. (1997). This interpretation of the compaction curve emphasizes the relationship between the geometry of the r.e.v. and the value of $\Sigma_{v}^{\prime}$. It explains the increase of $\Sigma_{v}^{\prime}$ observed as the compaction proceeds by the progressive closure of the cavity. Hence, if the geometry change at the microscopic level is neglected, the limit load is found to be constant and the compaction curve reaches an asymptote, as in the small strain plasticity simulation. 
It can be concluded that the comment formulated at Section 3.4 is valid also in this example: even for an infinitesimal macroscopic compaction, the micro-macro simulation of the macroscopic behavior must be performed within the framework of large strain analysis.

\subsection{Finite Poroplasticity: a Thermodynamic Approach}

To finish with, we summarize the main results of a thermodynamic approach of the skeleton constitutive law (Bourgeois et al., 1997). From now on, all physical quantities are defined at the macroscopic scale.

First, we extend the concept of unstressed configuration to porous media. It is assumed that the total stress and the pore pressure applied to the r.e.v. $\Omega_{t}$ can be set to zero in a reversible evolution. An unstressed configuration $\Omega_{U}$ is thus obtained. The deformation gradient $F$ of the skeleton is split into a plastic part $P$ which transforms $\Omega_{0}$ into $\Omega_{U}$, and an elastic part $E$ which transforms $\Omega_{U}$ into $\Omega_{t}$ (Fig. 7). The orientation of the unstressed configuration is fixed by the condition that $E$ be a symmetrical tensor. Besides, it is assumed that the elastic deformation gradient $E$ defines an infinitesimal (reversible) transformation:

$$
E=1+\mathcal{E}^{e} ;\left\{\begin{array}{l}
\left|\mathcal{E}^{e}\right|<1 \\
{ }^{t} \boldsymbol{\mathcal { E }}^{e}=\boldsymbol{E}^{e}
\end{array}\right.
$$

As in the theory of poroelasticity, an additional state variable is necessary in order to describe the volume change of the porous space. It is convenient to introduce the irreversible volume change $\phi^{p}\left|\Omega_{0}\right|$ and the reversible volume change $\phi^{e}\left|\Omega_{U}\right|$ which respectively occur during the plastic and elastic components of the transformation:

$$
F=E \cdot P ; \phi-\phi_{0}=\phi^{p}+\phi^{e} J^{p} \text { with } J^{p}=\operatorname{det} P
$$

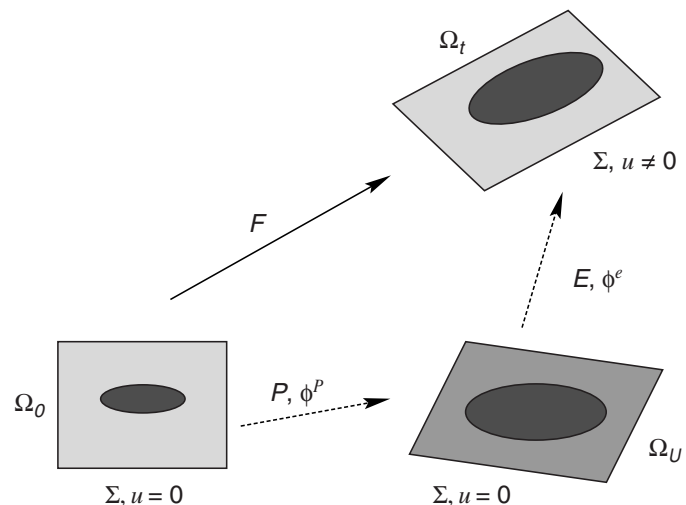

Figure 7

Decomposition of the skeleton transformation
We now look for the state equations of poroplasticity at large strains. As opposed to the case of an elastic solid constituent, the work $P_{e x t}$ of the external forces provided to the skeleton particle is partly dissipated. The dissipated energy is denoted by $\boldsymbol{D}\left|\Omega_{0}\right|$. The nondissipated energy is stored in the free energy $\Psi^{s k}\left|\Omega_{0}\right|$ of the skeleton particle. The expression of $P_{e x t}$ being the same as in Equation (20), the Clausius-Duhem inequality writes:

$$
\boldsymbol{D}=u \dot{\phi}+J \Sigma: D-\dot{\Psi}^{s k} \geq 0 \text { with } D=\left(\dot{F} \cdot F^{-1}\right)_{s}
$$

Using the decomposition (59) of $F$, the Eulerian strain rate $D$ can be split as in Equation (48). The corresponding expression of the dissipation is:

$$
\boldsymbol{D}=u \dot{\phi}+J \Sigma: \dot{E} \cdot E^{-1}+J \Sigma: E \cdot \dot{P} \cdot P^{-1} \cdot E^{-1}-\dot{\Psi} s k
$$

We first consider a particular elastic evolution in which $\dot{P}=0$. As there is no dissipation, Equation (61) takes the form:

$$
\dot{\Psi}^{s k}=J^{p} u \dot{\phi}^{e}+J^{p} \Sigma: \dot{\mathcal{E}}^{e}
$$

in which the assumption of small elastic strain (58) has been used in order to justify the approximations $J \approx J^{p}$ and $\dot{E} \cdot E^{-1} \approx \dot{\mathcal{E}}^{e}$. For the same reason as in Section 4.1.2, we now assume that the elastic properties of the skeleton are isotropic. This allows to consider the free-energy density $\dot{\Psi}^{s k}$ as a function of $\varepsilon^{e}, \phi^{e}$ and, if required, of a hardening parameter which is represented in the sequel by the plastic strain $\Delta^{p}$. With this assumption, the state equations of poroplasticity at large strains are immediately derived from Equation (62):

$$
J^{p} \sum=\frac{\partial \Psi^{s k}}{\partial \mathcal{E}^{e}} ; J^{p} u=\frac{\partial \Psi^{s k}}{\partial \phi^{e}}
$$

In the particular case of linear elastic (isotropic) properties in which $\Psi^{\mathrm{sk}}\left(\mathcal{E}^{e}, \phi^{e}, \Delta^{p}\right)$ is a quadratic function of the elastic state variables, Equation (63) can be put in the following form:

$$
\left\{\begin{array}{c}
J^{p} \sum=C_{0}: \mathcal{E}^{e}-b^{e} J^{p} u \\
J^{p} u=M\left(\phi^{e}-b^{e} \operatorname{tr} \mathcal{E}^{e}\right)
\end{array}\right.
$$

where $C_{0}$ is the tensor of macroscopic elastic moduli in drained conditions, $b^{e}$ and $M$ being scalar coefficients. These elastic parameters may a priori depend on $\Delta^{p}$. We note that Equation (64) introduces an effective stress $J^{p}\left(\Sigma+b^{e} u 1\right)$ which only depends on the value of the elastic strain $\mathcal{E}^{e}$. In the case of an incompressible solid, in which $J^{p}=b^{e}=1$, this result is consistent with the conclusions of Section 4.2.1. 
We now consider an arbitrary elastoplastic evolution in which $\varepsilon$ and $P \neq 0$. The (isotropic) state equations (63) allow to simplify the general expression (61) of the dissipation:

$$
\boldsymbol{D}=u \dot{\phi}^{p}+\sum: J^{p}\left(\dot{P} \cdot P^{-1}\right)_{s}-\frac{\partial \Psi^{s k}}{\partial \Delta^{p}}: \dot{\Delta}^{p}
$$

This expression is similar to the corresponding one in infinitesimal poroplasticity (Coussy, 1997), the classical plastic strain rate being here replaced by $J^{p}\left(\dot{P} \cdot P^{-1}\right)$. I identifies the pore pressure and the Cauchy stress as the thermodynamic forces respectively associated with the plastic porosity change and the plastic strain. This leads to write the flow rule, as in infinitesimal poroplasticity, with the help of a plastic potential $\boldsymbol{G}\left(\Sigma, u, \Delta^{p}\right)$ :

$$
\begin{aligned}
& J^{p}\left(\dot{P} \cdot P^{-1}\right)_{s}=\dot{\lambda} \frac{\partial \boldsymbol{G}}{\partial \Sigma}\left(\Sigma, u, \Delta^{p}\right) ; \\
& \dot{\phi}^{p}=\dot{\lambda} \frac{\partial \boldsymbol{G}}{\partial u}\left(\Sigma, u, \Delta^{p}\right) \text { with } \dot{\lambda} \geq 0
\end{aligned}
$$

If the plastic potential $\boldsymbol{G}$ depends on $\Sigma$ and $u$ through Terzaghi's effective stress $\Sigma+u 1$, Equation (66) show that:

$$
J^{p} \operatorname{tr} \dot{P} \cdot P^{-1}=\dot{\phi}^{p}
$$

The left- and righthand sides in Equation (67) respectively represent the volume change of the r.e.v. and the pore volume change during the plastic transformation. At the microscopic scale, Equation (67) thus implies that there is no volume change of the solid domain. Hence, the relevancy of Terzaghi's effective stress as regards the plastic potential and the flow rule is associated with the fact that the solid is plastically incompressible. Hence, the state equations (see Eq. (64)) and the flow rule a priori refer to two differen effective stress concepts.

\section{CONCLUSION}

The constitutive law of a solid subjected to large strains cannot be formulated in terms of a relationship between the Cauchy stress (or stress rate) and the strain (or strain rate). In the elastic case, the Piola-Kirchhoff stress is related to the Green-Lagrange strain. In the elastoplastic case, under some assumptions, the state equation relates the Jaumann derivative of the Cauchy stress to the difference between the total strain rate and the plastic strain rate.
Some aspects of the skeleton constitutive law at large strain can be clarified with the help of micro-macro techniques. In particular, the validity of Terzaghi's effective stress principle proves to be associated with solid incompressibility.

Even if the macroscopic strain applied to a r.e.v. is infinitesimal, the corresponding strain field at the microscopic level may be noninfinitesimal. Accordingly, in this case, the determination of the macroscopic behavior with the help of a micro-macro simulation should be performed within the framework of large strains.

In addition to the skeleton Green-Lagrange strain, the formulation of the skeleton constitutive law requires a second state variable, for instance the Lagrangian porosity, in order to take the volume change of the porous space into account. The pore pressure is the corresponding thermodynamic force.

\section{REFERENCES}

Bourgeois, E., de Buhan, P., Dormieux, L. (1997) Elastoplastic Porous Media at Large Strains: Thermodynamic Approach of the Constitutive Law and Numerical Resolution. In Proceedings of Complas V, Barcelone, 901-906.

Coussy, O. (1995) Mechanics of Porous Continua. Wiley.

De Buhan, P., Dormieux, L., Maghous, S. (1997) Analyse mécanique de l'effet de la compaction d'une couche géologique sur la réduction de porosité du matériau sédimentaire. In Proceedin

De Buhan, P. (1998) Private communication.

De Buhan, P., Chateau, X., Dormieux, L. (1998) The Constitutive Equations of Finite Strain Poroelasticity in the Light of a MicroMacro Approach. Eur. J. Mech. A/Solids, 17, 901-921.

Dienes, J.K. (1979) On the Analysis of Rotation and Stress Rate in Deforming Bodies. Acta Mechanica, 32, 217-323.

Gilormini, P. (1994) Sur les référentiels objectifs locaux en mécanique des milieux continus. C.R. Acad. Sci. Paris, 318, Série II, 1153-1159.

Lee, E.H. (1969) Elastic-Plastic Deformation at Finite Strains. J. Appl. Mech., 36, 1-6.

Mandel, J. (1971) Plasticité classique et viscoplasticité. CISM, Udine, Springer-Verlag. 